https://doi.org/10.15407/ujpe65.2.130

Z.M. LIASHENKO, ${ }^{1}$ I.A. LYASHENKO ${ }^{1,2}$

${ }^{1}$ Sumy State University

(2, Rimskii-Korsakov Str., Sumy 40007, Ukraine; e-mail: zinaida.makukha@gmail.com)

2 Technische Universität Berlin, Institut für Mechanik, FG Systemdynamik und Reibungsphysik (Sekr. C8-4, Raum M 122, Strasse des 17. Juni 135, Berlin 10623, Germany;

e-mail: i.liashenko@tu-berlin.de)

\title{
INFLUENCE OF SPATIAL INHOMOGENEITY ON THE FORMATION OF CHAOTIC MODES AT THE SELF-ORGANIZATION PROCESS
}

\begin{abstract}
The Lorentz system of equations, in which gradient terms are taken into account, has been solved numerically. Three fundamentally different modes of evolution are considered. In the first mode, the spatial distribution of the order parameter permanently changes in time, and domains of two types with positive and negative order parameter values are formed. In the second mode, the order parameter distribution is close to the stationary one. Finally, in the third mode, the order parameter is identical over the whole space. The dependences of the average area of domains, their number, and their total area on the time are calculated in the first two cases. In the third case, the contribution of gradient terms completely vanishes, and a classical Lorenz attractor is realized.

Ke ywords: self-organization, Lorenz system, strange attractor, order parameter, partial differential equations.
\end{abstract}

\section{Introduction}

Self-organization is a process of ordered structure formation at which the structure-forming parameter is not changed, i.e., when a specific external influence is absent [1]. We are faced with the self-organization processes in our daily life, both in animate and inanimate nature. Bright examples of self-organization include the ordering of electrons at low temperatures (superconductivity) [2], coherent laser radiation [1,3], the emergence of ordered vortex fluxes in the heated liquid, self-organization in nanomaterials (allotropic forms of carbon) [4], some chemical reactions (e.g., the Belousov-Zhabotinsky reaction) [5], the formation of ordered structures in viscous liquids [6], and so forth. In biological systems, the self-organization reveals itself as the coordinated behavior of bacteria, formation of ocean pyrosomes (these are large colonies of tiny organisms that look like hollow tubes closed at their one end), grouping of birds and fishes into flocks, and so on. Self-organization phenomena are observed very often in various complicated systems $[7,8]$.

(C) Z.M. LIASHENKO, I.A. LYASHENKO, 2020
The functioning of any living organism is also a permanent self-organization process. For example, a living cell is a very complicated system with channels for delivering nutrients and protein components, power plants (mitochondria), workshops for protein synthesis, and many other important components. By its structure, a cell resembles a megapolis in which the life is in full swing, but, seemingly, only at the expense of internal forces and without any external factors. Rather complicated self-organization processes that run in the cell can be observed today by simulating them. However, plenty of relevant issues still remain beyond the analysis.

According to the second thermodynamic law, the entropy can only increase for closed systems, i.e. the degree of their ordering always decreases in time. However, if we take a subsystem with a nonlinear behavior and provide it with an energy supply, the "excess" energy will be dissipated and afterward removed from the system. It is known that such local systems are capable of spontaneous ordering. One of the trivial examples is our Earth, on which life has spontaneously emerged. The Earth obtains energy from the Sun and emits it back into the space. A condition al-

ISSN 2071-0194. Ukr. J. Phys. 2020. Vol. 65, No. 2 
lowing a self-organization process to take place for a long time consists in an approximate equality between the input and output energy fluxes (in the case of the Earth, the latter should not be monotonically heated up or cooled down). From the aforesaid, it follows that there are stationary nonequilibrium processes in self-organized systems. Such systems comprise a subject of the synergetic science, with Hermann Haken being considered as its founder $[1,7]$.

Nowadays, there are plenty of relatively simple mathematical models for the description of self-organization processes in various physical systems. This is a well-known predator-prey model by Lotka and Volterra, the systems of Lorenz and Rössler differential equations that demonstrate chaotic attractors, various equations of chemical reactions, models of neural networks, and others. As a rule, those models are described by nonlinear equations with positive (the self-organization origin) and negative (the stabilization condition) feedbacks among dynamic variables.

In this work, we will focus attention on the wellknown Lorenz system [9], which is more than 50 years old, but still remains to be actively researched owing to the computer technology development. In particular, the Lorentz system of equations has been actively used for many years by the scientific group of O.I. Olemskoi, when describing the nonequilibrium noise-induced phase transitions [10], self-organization in quasiequilibrium condensation processes [11], kinetics of the first- and second-order phase transitions [12], and mechanisms of explosive crystallization [13] and self-organized criticality [14]. Furthermore, the Lorentz system is used to describe the chaotic radiation emission in lasers [3], fluxes in fluids, effects in electrical circuits in the presence of nonlinear elements (like Gunn diodes), chemical reactions [5], and so forth. When developing the relevant theories, the Lorentz system has been thoroughly analyzed with regard for the terms that are responsible for additive and multiplicative noises, nonlinear relaxation terms, a periodic external influence, and other factors. However, the insufficient attention has been paid to studying systems with spatially distributed parameters. For instance, some of such works were devoted to the analysis of the inhomogeneous melting of a lubricant in the contact zone between atomically smooth hard surfaces $[15,16]$. But the consideration was concentrated on the modes in which a single or- der parameter value was always established in time over the whole contact area, i.e. the system became homogenized. In this work, we will consider the case where the spatial distribution of the order parameter permanently changes in the course of system's evolution.

\section{Classical Lorenz Attractor}

The classical Lorenz system of equations looks like [9]

$\dot{X}=\sigma(Y-X)$,

$\dot{Y}=X(\rho-Z)-Y$,

$\dot{Z}=X Y-\beta Z$.

The dotted variables stand for their derivatives with respect to the time $t^{\prime}$, e.g., $\dot{X} \equiv d X / d t^{\prime}$. The variables $X, Y$, and $Z$, as well as the constants $\sigma, \rho$, and $\beta$, may have different physical contents depending on the problem described by Eqs. (1)-(3). For example, when describing a single-mode laser, $X$ is the wave amplitude in a laser resonator, $Y$ the polarization magnitude, $Z$ the inversion of the energy level population, the constants $\beta$ and $\sigma$ describe the ratio between the relaxation coefficients of the indicated quantities, and the parameter $\rho$ is the pump intensity. Of all those parameters for a particular physical system with given characteristics, we can only change the parameter $\rho$, which is an external control one.

A specific feature of model (1)-(3) consists in that it is possible to realize various operating modes of the system concerned, by changing the parameter $\rho$. This can be either the relaxation mode or the mode of deterministic chaos, when the system behavior is characterized by a strange attractor. System (1)-(3) is the simplest one that makes it possible to represent a selforganization process. Therefore, its study is of interest even without a connection to a definite physical phenomenon.

Equations (1)-(3) can be simplified by introducing the new variables,

$t \equiv \sigma t^{\prime} ; \quad u \equiv X / \sqrt{\beta} ; \quad v \equiv Y / \sqrt{\beta}$

$S \equiv \rho-Z ; \quad \delta \equiv \sigma / \beta ; \quad S_{e} \equiv \rho$,

where $t^{\prime}$ and $t$ denote the time in the initial and transformed systems, respectively. In terms of the new variables, the Lorentz system of equations looks like

$\dot{u}=-u+v$,

$\sigma \dot{v}=-v+S u$, 

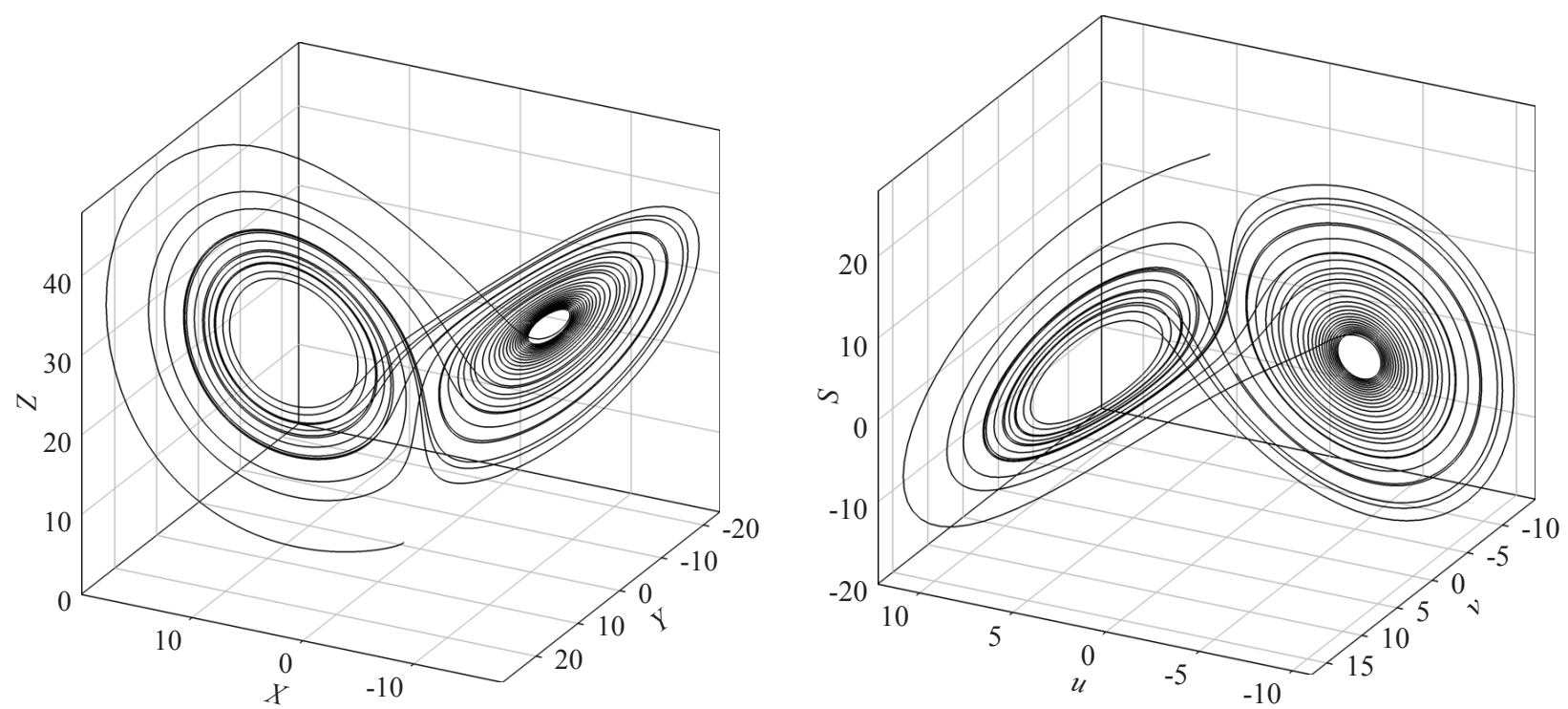

Fig. 1. Attractors obtained by solving the initial system of equations (1)-(3) (left panel) and modified system of equations (5)-(7). The left panel: the parameters $\sigma=10, \rho=28, \beta=8 / 3$, and the initial conditions $X_{0}=Y_{0}=Z_{0}=1$; the right panel: the parameters $\sigma=10, \delta=3.75, S_{e}=2810$, and the initial conditions $u_{0}=v_{0}=\sqrt{0.375}, S_{0}=27$

$\delta \dot{S}=\left(S_{e}-S\right)-u v$.

In this form, it was analyzed in works [10-14]. The system of equations (1)-(3) reveals a strange attractor mode at the parameter values $\sigma=10, \rho=28$, and $\beta=8 / 3$, which, according to expressions (4), correspond to the values $\sigma=10, S_{e}=28$, and $\delta=3.75$ for the modified system (5)-(7). Hence, only the value of the last parameter changes, and this value can be written as an exact decimal fraction in the given representation.

If the Lorentz system of equations is solved numerically, the choice of initial conditions is very important, because they substantially affect the final result. Let the initial conditions for the system of equations (5)-(7) be equal to $u_{0}, v_{0}$, and $S_{0}$, respectively. This, according to relations (4), gives the initial conditions $X_{0}=u_{0} \sqrt{\sigma / \delta}, Y_{0}=v_{0} \sqrt{\sigma / \delta}$, and $Z_{0}=S_{e}-S_{0}$ for the system of equations (1)-(3). In order to provide a full consistency of the results of a numerical simulation obtained when solving the initial, Eqs. (1)-(3), and modified, Eqs. (5)-(7), systems, we have also to take into account that the time $t$ is measured in different units in them (see Eqs. (4)). If a solution of the initial system is sought in the time interval $t^{\prime} \in[0, T]$, the equivalent time interval for the modified system equals $t \in[0, \sigma T]$.
It is worth noting that, in the strange-attractor mode, the further behavior of the system is affected even by a minor change in the initial conditions or by the unavoidable errors of a numerical calculation. Therefore, even if the step of integration $\Delta t^{\prime}$ is relatively small, there is a fixed time after which system's behavior begins to deviate from that obtained at the solution of equations with a smaller step. Therefore, in order to provide the total correspondence between the obtained results, different integration steps over the time must be selected for each system in addition to the initial conditions described above. For example, if the time step to integrate system (1)-(3) is chosen to equal $\Delta t^{\prime}$, then, according to expressions (4), the step $\Delta t=\sigma \Delta t^{\prime}$ has to be taken for the modified equations (5)-(7).

Figure 1 demonstrates the results of a numerical solution of the systems of equations (1)-(3) (left panel) and (5)-(7) (right panel). The set of initial conditions $X_{0}=Y_{0}=Z_{0}=1$ was selected in the former case, which, according to Eqs. (4), corresponded to the set $u_{0}=v_{0}=\sqrt{0.375}$ and $S_{0}=27$. The equations were solved using the Euler method with the time increments $\Delta t^{\prime}=10^{-8}$ and $\Delta t=10^{-7}$. At the selected parameter values and initial conditions, the dependences shown in Fig. 1 exactly reproduce the same process. Furthermore, since the parameters

ISSN 2071-0194. Ukr. J. Phys. 2020. Vol. 65, No. 2 
in the both systems are linearly interdependent [see Eqs. (4)], there are no qualitative differences between the panels.

In the next section, we will analyze the modified system of equations (5)-(7). Note that all results obtained below can be interpreted proceeding from the initial Lorentz system (1)-(3).

\section{Account for a Spatial Inhomogeneity and the Numerical Calculation Procedure}

In order to describe an inhomogeneous distribution of parameters, system (5)-(7) should include the additional gradient terms $D_{u} \nabla^{2} u, D_{v} \nabla^{2} v$, and $D_{S} \nabla^{2} S$. Let the spatial coordinates be denoted as $x^{\prime}$ and $y^{\prime}$. Then the operator $\nabla^{2}$ is written as $\nabla^{2}=$ $=d^{2} / d x^{\prime 2}+d^{2} / d y^{\prime 2}$ in the two-dimensional case. After introducing the new spatial variables $x=x^{\prime} / \sqrt{D_{S}}$ and $y=y^{\prime} / \sqrt{D_{S}}$, the equations with gradient terms acquire the following final form:

$\dot{u}=D_{(u S)} \nabla^{2} u-u+v$,

$\sigma \dot{v}=D_{(v S)} \nabla^{2} v-v+S u$,

$\delta \dot{S}=\nabla^{2} S+\left(S_{e}-S\right)-u v$,

where $D_{(u S)}=D_{u} / D_{s}$ and $D_{(v S)}=D_{v} / D_{s}$.

To solve the system of equations (8)-(10) numerically, let us divide each of the spatial coordinate axes $x$ and $y$, as well as the time axis $t$, into a certain number of identical segments. Then the solution of the problem in the whole space-time region is reduced to the determination of the values at the nodes of a three-dimensional finite-difference mesh. Let $n, j$, and $k$ be the ordinal numbers of a mesh node reckoned along the axes $t, x$, and $y$, respectively, and let those numbers acquire the following values: $n=0,1,2, \ldots, M ; j=1,2,3, \ldots, N_{x}$; and $k=1,2,3, \ldots, N_{y}$. With the help of this notation, the values of the variables $t, x$, and $y$, at the $(n, j, k)$-th node of the space-time mesh are given by the expressions $t^{n}=n \Delta t, x_{j}=(j-1) H_{x}$, and $y_{k}=(k-1) H_{y}$, respectively, where $\Delta t$ is the previously introduced mesh step along the time axis, whereas $H_{x}$ and $H_{y}$ are the mesh steps along the $x$ and $y$ axes, respectively. We used an explicit finite-difference scheme in the numerical simulation. Therefore, the derivatives in the system of equations were approximated as follows [17]:

$\frac{\partial u}{\partial t}=\frac{u_{j, k}^{n+1}-u_{j, k}^{n}}{\Delta t} ;$

ISSN 2071-0194. Ukr. J. Phys. 2020. Vol. 65, No. 2 $\begin{aligned} \frac{\partial^{2} u}{\partial x^{2}} & =\frac{u_{j+1, k}^{n}-2 u_{j, k}^{n}+u_{j-1, k}^{n}}{H_{x}^{2}} ; \\ \frac{\partial^{2} u}{\partial y^{2}} & =\frac{u_{j, k+1}^{n}-2 u_{j, k}^{n}+u_{j, k-1}^{n}}{H_{y}^{2}} .\end{aligned}$

When substituting expressions (11) into system (8)-(10), we obtain an explicit finite-difference scheme. Provided that the initial and boundary conditions are given (see below), this scheme has a single unknown quantity $u_{j, k}^{n+1}$. Expressing this quantity from a difference scheme that corresponds to the basic equations and putting $H_{x}=H_{y}=H$ for simplicity, we obtain

$u_{j, k}^{n+1}=u_{j, k}^{n}+\frac{D_{(u S)} \Delta t}{H^{2}}\left(u_{j+1, k}^{n}+u_{j, k+1}^{n}-4 u_{j, k}^{n}+\right.$

$\left.+u_{j, k-1}^{n}+u_{j-1, k}^{n}\right)+\Delta t\left(-u_{j, k}^{n}+v_{j, k}^{n}\right)$,

$v_{j, k}^{n+1}=v_{j, k}^{n}+\frac{D_{(v S)} \Delta t}{\sigma H^{2}}\left(v_{j+1, k}^{n}+v_{j, k+1}^{n}-4 v_{j, k}^{n}+\right.$

$\left.+v_{j, k-1}^{n}+v_{j-1, k}^{n}\right)+\frac{\Delta t}{\sigma}\left(-v_{j, k}^{n}+S_{j, k}^{n} u_{j, k}^{n}\right)$,

$S_{j, k}^{n+1}=S_{j, k}^{n}+\frac{\Delta t}{\delta H^{2}}\left(S_{j+1, k}^{n}+S_{j, k+1}^{n}-4 S_{j, k}^{n}+S_{j, k-1}^{n}+\right.$

$\left.+S_{j-1, k}^{n}\right)+\frac{\Delta t}{\delta}\left(S_{e}-S_{j, k}^{n}-u_{j, k}^{n} v_{j, k}^{n}\right)$.

For the numerical implementation of the obtained procedure (12)-(14), the corresponding initial and boundary conditions have to be determined. The initial conditions are given using the Box-Muller function [18]

$u_{j, k}^{0}=\sqrt{2 I} \sqrt{-2 \ln r_{1}} \cos \left(2 \pi r_{2}\right)$,

where $r_{1}$ and $r_{2}$ are pseudorandom numbers with a uniform distribution within the interval $(0,1]$, and $I$ is the stochastic source intensity, which determines the dispersion $\mu=\sqrt{2 I}$. The initial distributions for the quantities $u_{j, k}^{0}$ and $S_{j, k}^{0}$ are chosen in a similar way. As a result, the initial spatial distributions are Gaussian,

$P(\xi)=\frac{1}{\sqrt{2 \pi} \mu} \exp \left\{-\frac{\xi^{2}}{2 \mu^{2}}\right\}$

for all three quantities $\xi=u, v, S$.

When solving Eqs. (12)-(14), the corresponding boundary conditions have to be selected proceeding from the physical underground of the problem. For 


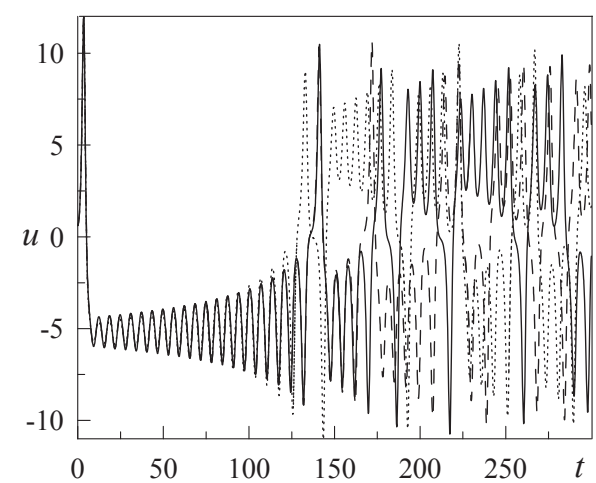

Fig. 2. Dependences $u(t)$ obtained by numerically solving the system of equations (5)-(7) and corresponding to the parameters in the right panel of Fig. 1

the sake of simplification, let us consider the infinite surfaces and neglect the boundary effects. For this purpose, let us apply periodic boundary conditions. In so doing, only four conditions have to be taken into account in Eqs. (12)-(14). Namely, if $j=1$, then $j-1 \rightarrow N_{x}$; if $k=1$, then $k-1 \rightarrow N_{y}$; if $j=N_{x}$, then $j+1 \rightarrow 1$; and if $k=N_{y}$, then $k+1 \rightarrow 1$.

In order to solve the inhomogeneous system of equations described above, it is necessary to properly select the discretization parameters: $\Delta t$ for the time axis, and $H$ for the spatial region. As was already mentioned, the Lorentz system is very sensitive to the choice of the initial conditions and to the unavoidably emerging errors of numerical calculations. For example, in Fig. 2, the dependences $u(t)$ obtained by solving Eqs. (12)-(14) are shown. Here, the solid curve corresponds to the parameters in the right panel in Fig. 1, including the time integration step $\Delta t=10^{-7}$; the dashed curve corresponds to the value $\Delta t=10^{-5}$, and the dotted one to $\Delta t=10^{-2}$. In other cases, all parameters are identical, including the initial conditions.

The solid curve demonstrates the most accurate result, because it was obtained with the minimum value of the time integration step. Two other curves begin to deviate from the solid one at definite time moments, when the corresponding accumulated errors of the numerical calculation become rather large. The smaller the $\Delta t$ value, the earlier this deviation begins. Hence, the conclusion can be drawn that, for the trajectory to be calculated accurately, it has to be calculated several times with the shorter and shorter time discretization step $\Delta t$. The obtained dependences are used to determine a region, where the trajectories overlap, and this time interval will correspond to the exact solution of the system.

But, under real conditions, there are always fluctuations and external uncontrolled factors of various kinds that prevent such complicated systems from manifesting a deterministic behavior, even if the initial conditions are the same. Therefore, the exact numerical solution of the Lorentz system of equations is of no interest, because the behavior of the strangeattractor type is a more important characteristic in this case. As one can see from Fig. 2, the strange Lorenz attractor is realized for all selected $\Delta t$ values, so that it is enough to choose the time step $\Delta t=10^{-5}$ for a numerical simulation. The explicit finite-difference scheme (11) is stable only conditionally. It provides a stable solution, if the coefficients before the approximations of coordinate derivatives in Eqs. (12)-(14) are less than 0.5 [17]. In particular, for Eq. (12), this condition looks like $D_{(u S)} \Delta t / H^{2}<0.5$. In what follows, the discretization parameters will be chosen, taking the stability conditions into account.

\section{Simulation Results and Their Discussion}

For the simulation, we took the discretization parameter values $\Delta t=10^{-5}$ and $H=0.01$. The solution was sought on a spatial $256 \times 256$-mesh. Taking the discretization parameters into account, the size of a mesh square amounted to $2.56 \times 2.56$. The same parameter values $S_{e}=28, \sigma=10$, and $\delta=3.75$ were selected in all cases considered below. The system of equations was solved numerically (see Section 3) within the time interval $t \in[0,1000]$. The intensity of a stochastic source in function (15) for the formation of initial conditions was chosen to equal $I=10$. The set of initial conditions was generated only once, and it was used afterward in all cases. In the course of the solution procedure, a map of the order parameter values $u(x, y)$ and the average value $\langle u\rangle$ were regularly stored.

Three cases of relations among the coefficients responsible for the gradient contribution were considered: (i) $D_{u S}=D_{v S}=10^{-3}$, (ii) $D_{u S}=D_{v S}=10^{-2}$, and (iii) $D_{u S}=D_{v S}=10^{-1}$. The results obtained are depicted in Fig. 3. One can clearly see that they correspond to three essentially different modes. In the first case (upper panel), a mode is established in which the spatial distribution of the order parameter and, accordingly, its average value permanently

ISSN 2071-0194. Ukr. J. Phys. 2020. Vol. 65, No. 2 
change. The second case (middle panel) corresponds to a situation where a certain structure close to the stationary one is formed, and the average value of the order parameter changes rather weakly in time. Finally, the third case (bottom panel) illustrates a mode, where the influence of gradient contributions is negligible. As one can see, the latter dependence reproduces the mode described in Fig. 2, which is established, if the gradient terms are not taken into account.

It should be noted that, in the first two cases, the mean order parameter value changes within much narrower intervals than in the third case. This effect can be a result of either a certain homogenization in the system induced by the gradient terms or the effect of phase separation, when there emerge islands on the plane with negative and positive order parameter values. In the latter case, the time dependences of the mean values contain no useful information about the system, and it is necessary to analyze the root-meansquare deviation ( $\mathrm{rms}$ ) from the mean value.

In order to determine the rms deviation of the order parameter, we apply the standard procedure,

$\left\langle u^{2}\right\rangle^{1 / 2}=\left\langle(u-\langle u\rangle)^{2}\right\rangle^{1 / 2}$

where the broken brackets mean the averaging over the coordinate region $(256 \times 256=65536$ values in total). The results obtained are exhibited in Fig. 4. In the case $D_{u S}=D_{v S}=10^{-3}$ (upper panel in Fig. 4), the quantity $\left\langle u^{2}\right\rangle^{1 / 2}$ varies within a much larger interval than the quantity $\langle u\rangle$ does in Fig. 3. This behavior can be explained by the formation of islands with different order parameter signs. The configuration of islands changes permanently in time at that, and the system does not tend to a certain stationary distribution of the order parameter. If $D_{u S}=D_{v S}=10^{-2}$ (middle panel), the almost stationary configuration of islands is established in the system. Nevertheless, it should be noted that a slow evolution in time is still observed (the inset in the middle panel). In the third case with $D_{u S}=D_{v S}=10^{-1}$ (lower panel), the system quickly transits into a mode in which the order parameter acquires the same value over the whole simulation region, so that the contribution of gradient terms completely vanishes according to Eq. (11). Hence, the analysis of the $\left\langle u^{2}\right\rangle^{1 / 2}$ dependences confirms the assumptions made above.

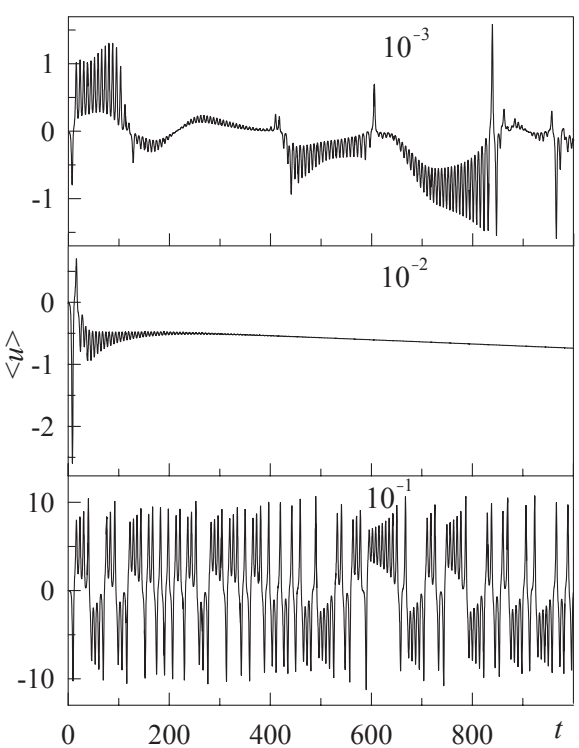

Fig. 3. Average order parameter value $\langle u\rangle$ as a function of the time $t$ for the parameter ratios $D_{u S}=D_{v S}=10^{-3}$ (upper panel), $10^{-2}$ (middle panel), and $10^{-1}$ (lower panel)

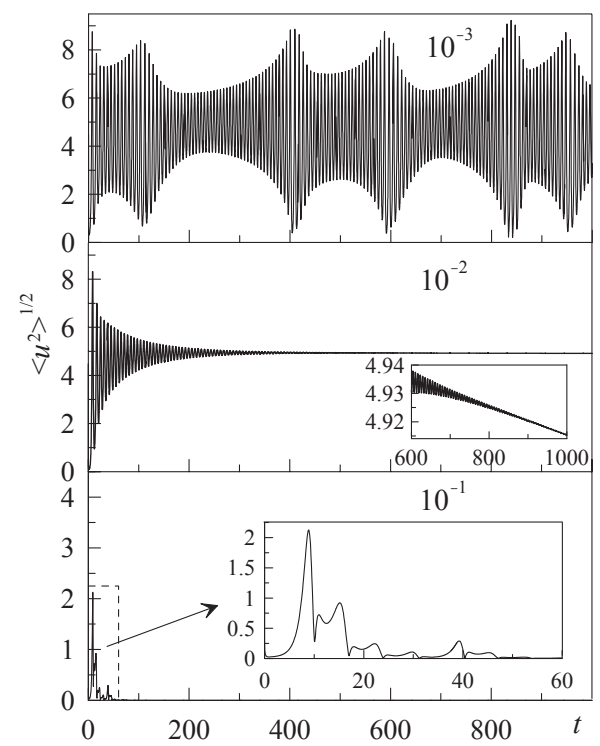

Fig. 4. Root-mean-square deviations $\left\langle u^{2}\right\rangle^{1 / 2}$ [Eq. (17)] of the order parameter from the mean value as functions of the time $t$, which correspond to the data of Fig. 3. The scaled-up fragments of the dependences are shown in the insets

The obtained distributions of the order parameter can be analyzed making use of the corresponding methods for three-dimensional surfaces. At present, there are two rather powerful methods used for surface research: the multifractal fluctuation analysis 


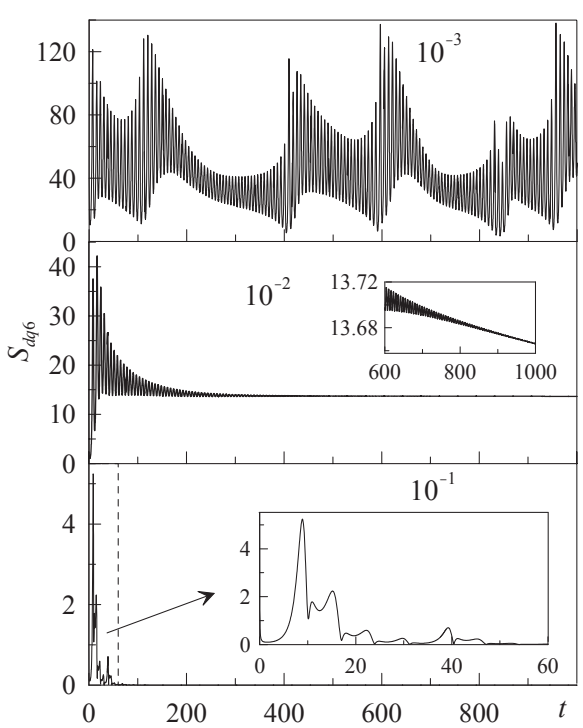

Fig. 5. Root-mean-square slope $S_{d q 6}$ calculated by formulas (19) and (21) as a function of the time $t$. The parameters are the same as in Fig. 4. The scaled-up fragments of the dependences are shown in the insets

$[19,20]$ and the methods based on the analysis of the spectral power density [21]. At the same time, threedimensional surfaces are characterized by a variety of parameters that are calculated numerically [22]. In order to elucidate the influence of surface irregularities (the surface roughness) on the physical processes of interaction between the surfaces, a more complicated modeling is often required. For example, the boundary element method $[23,24]$ is actively used to calculate the adhesive properties of surfaces.

One of the important characteristics in the surface analysis is the mean-square deviation (Fig. 4). Another important parameter that characterizes surface irregularities is the rms slope $S_{d q}$, which is calculated according to the following formula ${ }^{1}$ :

$S_{d q}^{2}=\frac{1}{A} \iint\left[\left(\frac{\partial u(x, y)}{\partial x}\right)^{2}+\left(\frac{\partial u(x, y)}{\partial y}\right)^{2}\right] \mathrm{d} x \mathrm{~d} y$,

where $x$ and $y$ are the spatial coordinates, and $A$ is the area of a simulated surface (in our case, $A=$ $=2.56 \times 2.56$ square units). In effect, formula (18) gives the mean value of the derivative describing

\footnotetext{
1 Formulas (18) and (19) are given for $S_{d q}^{2}$ to avoid the radical sign in the right-hand sides of the formulas. In calculations, the parameter $S_{d q} \equiv\left(S_{d q}^{2}\right)^{1 / 2}$ has to be determined.
}

\section{6}

the slope of surface irregularities over the whole surface. In the situation concerned, the maximum slope is at the domain boundaries, where the order parameter changes its sign. Therefore, the quantity $S_{d q}$ [Eq. (18)] can be used to indirectly characterize the number of domains. A discrete analog of formula (18) can be written in the form

$S_{d q}^{2}=\frac{1}{(M-1)(N-1)} \sum_{i=1}^{M-1} \sum_{j=1}^{N-1} B_{i j}$,

where

$$
\begin{aligned}
& B_{i j}=\left(\frac{u\left(x_{i}, y_{j}\right)-u\left(x_{i-1}, y_{j}\right)}{\Delta x}\right)^{2}+ \\
& +\left(\frac{u\left(x_{i}, y_{j}\right)-u\left(x_{i}, y_{j-1}\right)}{\Delta y}\right)^{2}
\end{aligned}
$$

$\Delta x$ and $\Delta y$ are the parameters of the spatial discretization $(\Delta x=\Delta y=H)$, and $M$ and $N$ are the numbers of points on the axes $(M=N=256)$.

Expression (19) is not the only formula used to determine the parameter $S_{d q}$. Another numerical scheme is often applied to approximate the derivatives over six neighbor points by replacing their discrete representations at the point $\left(x_{i}, y_{j}\right)$. In this case, another expression for $B_{i j}$ has to be applied in formula (19):

$B_{i j}=\left(\frac{A_{1}}{60 \Delta x}\right)^{2}+\left(\frac{A_{2}}{60 \Delta y}\right)^{2}$

where

$A_{1}=u\left(x_{i+3}, y_{j}\right)-9 u\left(x_{i+2}, y_{j}\right)+45 u\left(x_{i+1}, y_{j}\right)-$

$-45 u\left(x_{i-1}, y_{j}\right)+9 u\left(x_{i-2}, y_{j}\right)-u\left(x_{i-3}, y_{j}\right)$,

$A_{2}=u\left(x_{i}, y_{j+3}\right)-9 u\left(x_{i}, y_{j+2}\right)+45 u\left(x_{i}, y_{j+1}\right)-$

$-45 u\left(x_{i}, y_{j-1}\right)+9 u\left(x_{i}, y_{j-2}\right)-u\left(x_{i}, y_{j-3}\right)$.

Let us denote the rms slope $S_{d q}$ calculated with the help of approximation (21) by $S_{d q 6}$. The both definitions coincide with a high accuracy for relatively smooth surfaces. However, a significant difference between the corresponding values can take place, if the irregularities are large. Therefore, by comparing the results obtained using those two formulas, it is possible to estimate the smoothness of the examined surface.

ISSN 2071-0194. Ukr. J. Phys. 2020. Vol. 65, No. 2 

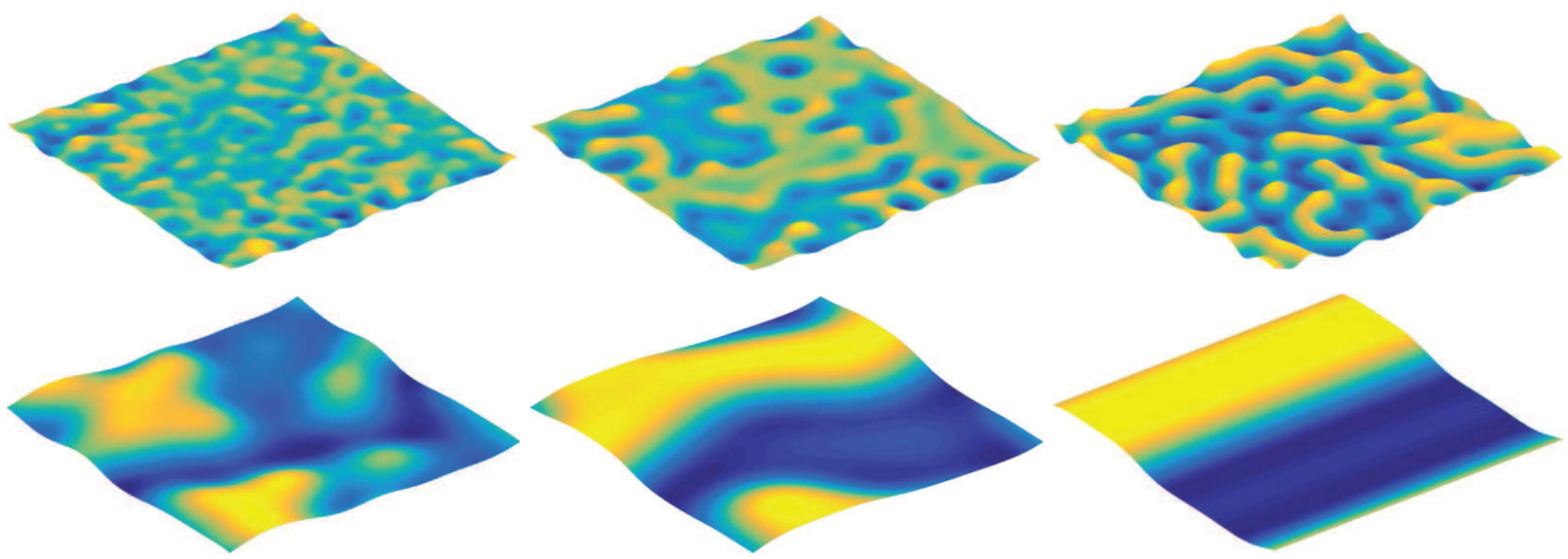

Fig. 6. Spatial distributions of the order parameter at $D_{u S}=D_{v S}=10^{-3}$ (upper panels) and $10^{-2}$ (lower panels). The time $t=10$ (left panels), 100 (middle panels), and 1000 (right panels)

In Fig. 5, the time dependences of the rms slope $S_{d q 6}$ calculated for three relations among the gradient terms are plotted. Qualitatively, the dependences almost reproduce the rms deviation of the order parameter in time (Fig. 4), but they also have some differences. For example, the rms deviation $\left\langle u^{2}\right\rangle^{1 / 2}$ in the case $D_{u S}=D_{v S}=10^{-2}$ deviates in two directions at the beginning of the motion, whereas the rms slope $S_{d q 6}$ rather quickly ceases to fall below a certain value close to the stationary one. For the parameter values $D_{u S}=D_{v S}=10^{-1}$, the dependences $\left\langle u^{2}\right\rangle^{1 / 2}(t)$ and $S_{d q 6}$ are qualitatively identical.

The specific features of all three considered cases remain unchanged: a permanent variation of the order parameter in time, a mode close to the stationary one, and a homogenized system, respectively. In the case of surfaces, for their complete characteristics, it is necessary to know both the rms deviation and the rms slope. The combination of those two parameters almost completely determines the properties of surfaces at their interaction. For example, when describing the adhesion of rough surfaces, the product $\left\langle u^{2}\right\rangle^{1 / 2} \times S_{d q 6}(t)$ is reduced to the Johnson parameter [25], which characterizes the adhesive strength of the contact.

Hence, according to the results obtained, a conclusion can be drawn that, for the parameter ratios $D_{u S}=D_{v S}=10^{-1}$, only the region of the dependence with the transition mode $(t \in[0,60])$ may be of interest, whereas the rest of the dependence is not, because the spatial structure becomes completely homogenized in time [16]. Let us consider two other

ISSN 2071-0194. Ukr. J. Phys. 2020. Vol. 65, No. 2 cases of the ratios among the coefficients $D$ in more details.

Figure 6 demonstrates the evolution of the spatial distributions of the order parameter $u$ in the cases $D_{u S}=D_{v S}=10^{-3}$ (upper panels) and $D_{u S}=$ $=D_{v S}=10^{-2}$ (lower panels). The time $t$ increases in the direction from left to right $(t=10,100$, and 1000). One can see that a stationary structure with two regions - namely, with positive and negative $u$ values - is formed in the case $D_{u S}=D_{v S}=10^{-2}$. In another case, $D_{u S}=D_{v S}=10^{-3}$, a permanent evolution of the order parameter distribution is observed. Let us refer to the regions with fixed order parameter signs as to positive or negative domains. For example, when describing the magnetization, the orientation of the magnetic moment vector does not change within such a domain. The examined Lorentz system of equations can be used to describe the evolution of the specimen magnetization, but, first, it must be properly parametrized. In particular, the deformation can be chosen as an external factor (parameter $S_{e}$ ), and the model will describe the dependence of the specimen magnetization on the external mechanical action. If the magnetic field is chosen as an external factor, then the evolution of the tensosensitivity coefficient can be traced [26]. Furthermore, spatial distributions of this kind take place in biological systems [27] and, generally speaking, in many dynamic processes. It should be noted that such distributions can be calculated using various methods. In particular, it can be the direct solution of evolution equations [27] or it can be more sophisticated 

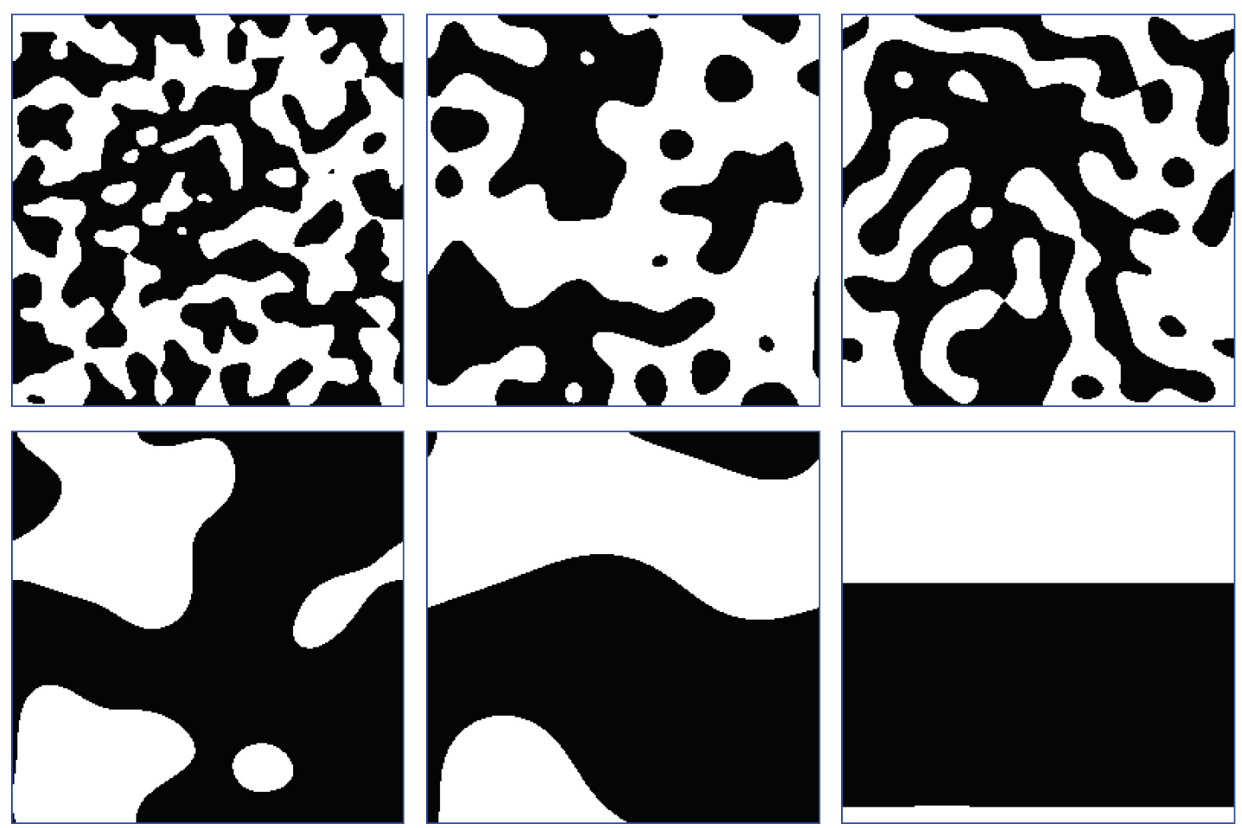

Fig. 7. Spatial distributions of the order parameter sign corresponding to the order parameter distributions shown in Fig. 6. White areas correspond to positive order parameter values, and black areas to negative ones

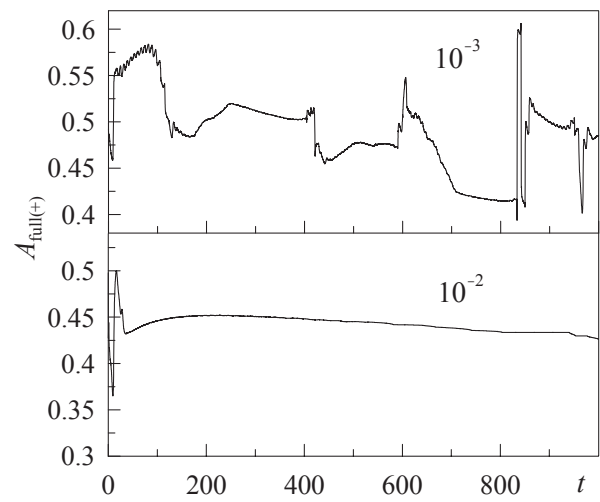

Fig. 8. Dependences of the total area of positive domains normalized by the system size, $A_{\text {full(+) }}$, on the time $t$ for $D_{u S}=D_{v S}=10^{-3}$ (upper panel) and $10^{-2}$ (lower panel)

methods, such as the methods of molecular dynamics [28].

In some problems, the magnitude of the order parameter is not very important, because the system behavior is governed only by the order parameter sign. In such cases, it is much simpler to analyze the distributions of the binary type, in which the order parameters of domains acquire values of 0 and 1 . The processing of such binary distributions takes much less CPU time and memory to store them on the hard disk. Visually, different order parameter characters can be represented by different colors. Figure 7 illustrates such binary distributions, which completely correspond to Fig. 6. The white color corresponds to positive domains, and the black color to negative ones.

Let us analyze the binary distributions of the order parameter depicted in Fig. 7. Recall that the spatial distributions were regularly stored with a certain periodicity during the whole time of the simulation. Figures 6 and 7 illustrate only a few of them to visualize different scenarios of the system behavior.

Figure 8 exhibits the time dependences of the summed-up areas of positive domains $A_{\text {full( }+)}(t)$ normalized to the total area of the system. The area $A_{\text {full(-) }}$ occupied by negative domains is not reflected here, because it can be easily calculated as $A_{\text {full(-) }}=$ $=1-A_{\text {full (+) }}$. From the upper panel of Fig. 8 , it follows that the domains of two types competitively interact with each other throughout the whole time interval, and the general configuration of the system is under a permanent reconstruction. A certain symmetry takes place at that, because the average total areas of the domains of both types are approximately 
identical in the course of system's evolution, which corresponds to the equivalent filling of the space by the domains of both types. At the initial time moment, $A_{\text {full(-) }} \approx A_{\text {full(+) }} \approx 0.5$, because the initial conditions were given by equiprobably selecting the sign of the parameter $u$ on the basis of formula (16). The lower panel in Fig. 8 illustrates a situation where the domain areas change much more slowly in comparison with the case $D_{u S}=D_{v S}=10^{-3}$ (upper panel in Fig. 8) ${ }^{2}$.

Now, let us analyze how the numbers of domains of both characters depend on the time. The corresponding dependences are shown in Fig. 9 on a loglog scale. A common feature in both cases is that the numbers of domains of both signs substantially diminish at the initial stage of system's evolution owing to the merging of small domains. Only after a rather long time period, the system behavior in those cases begins to differ considerably. In the upper panel of Fig. 9, the numbers of domains of both characters permanently vary during the whole simulation time interval.

The inset in the upper panel additionally illustrates changes in the domain numbers within the time interval $t \in[100,1000]$ on a linear scale for each axis. There is a certain correlation between the behaviors of two groups of domains. Namely, the number of domains with a definite sign increases together with the growth of the group of domains with the opposite sign, which is quite reasonable, because two domains with different signs are adjacent to each other.

In order to avoid misunderstandings, an important issue should be pointed out. The periodic boundary conditions were not taken into account when analyzing the images (e.g., the bottom right corner in Fig. 7). As a result, the program recognizes one negative and two positive domains in this situation. Such a seemingly illogical relationship between the numbers of domains takes place owing to the confinement of the system. The same calculation technique was used by us in work [16].

In the bottom panel of Fig. 9 (for $D_{u S}=D_{v S}=$ $=10^{-2}$ ), the system slowly evolves to a state with one negative and two positive domains (the bottom-

\footnotetext{
2 The scale spans of the ordinate axes in the upper and lower panels of Fig. 8 were selected to be identical and equal to 0.24 in order to visually compare how strongly the areas change with respect to each other.
}

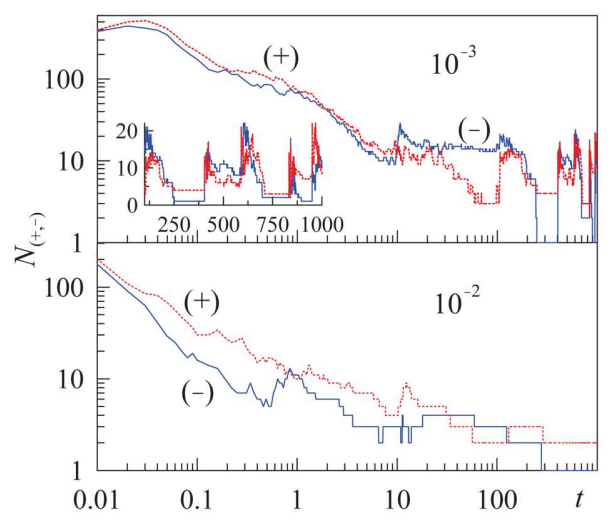

Fig. 9. Dependences of the number $N$ of domains with positive (dotted curves) and negative (solid curves) order parameter values on the time $t$ for $D_{u S}=D_{v S}=10^{-3}$ (upper panel) and $10^{-2}$ (lower panel)

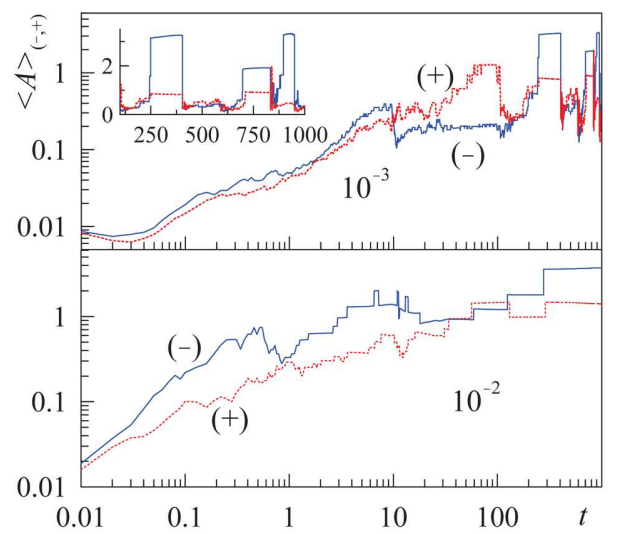

Fig. 10. Dependences of the average area $\langle A\rangle$ of domains with positive (dotted curves) and negative (solid curves) order parameter values on the time $t$ for $D_{u S}=D_{v S}=10^{-3}$ (upper panel) and $10^{-2}$ (lower panel)

right panel in Fig. 7). Nevertheless, in this case, the number of domains decreases much more rapidly in time. For instance, in the upper panel of Fig. 9, there are approximately 70 domains of both signs at the time $t=1$. On the other hand, there are only about 10 domains of each type at the same time moment in the lower panel.

The plots describing the variation of the mean domain area $\langle A\rangle$ for the domains of both types are exhibited in Fig. 10. From the qualitative viewpoint, these dependences are similar to the inverted dependences of the domain number $N$ on the time $t$ (Fig. 9). In both situations (upper and lower pan- 
els in Fig. 10), the average domain area increases with time, which can be explained by a reduction in the domain number. This process occurs simultaneously with the growth of the domain size. At $D_{u S}=D_{v S}=10^{-2}$ (lower panel), the domain area reaches an almost stationary value by the end of the simulation time, whereas the stationary mode is not attained, if $D_{u S}=D_{v S}=10^{-3}$ (upper panel; this is better illustrated in the inset).

The average area of domains belonging to a definite type was calculated as the ratio between the total area of domains of this type and their number. In other words, in effect, the dependences characterize the ratio between the total area of domains, $A_{\text {full }}$ (Fig. 8), and their number $N$ (Fig. 9). The difference consists in that Fig. 8 demonstrates the area normalized to the total system size, whereas Fig. 10 illustrates the absolute area value, where the total system area equals $256 \times 256 \times H^{2}=6.5536$ square units, and $H$ is the discretization parameter included into Eqs. (12)-(14).

\section{Analysis of the Fractal Properties of Order Parameter Distributions}

As was mentioned above, the multifractal fluctuation analysis and the methods in which the power spectral density $C(q)$ is analyzed [19-21] are rather powerful techniques of the surface research. The latter technique, in our opinion, is more universal, because it is based on a direct analysis of the spectral density dependence $C(q)$, which can be calculated for any surface, whereas the multifractal fluctuation analysis allows only the surfaces that possess fractal properties to be analyzed. However, the both methods give interesting results for fractal objects.

There are several definitions for the function $C(q)$. We will use the definition from work [21],

$C(\mathbf{q})=\frac{1}{(2 \pi)^{2}} \int \mathrm{d}^{2} x\langle h(\mathbf{x}) h(\mathbf{0})\rangle \mathrm{e}^{-i \mathbf{q} \mathbf{x}}$,

where $h$ is the altutude of surface points, $\mathbf{x}$ is the radius vector $(|\mathbf{x}| \equiv x)$, and $\mathbf{q}$ is the wave vector. When calculating the dependence $C(q)$, it is convenient to use the built-in MATLAB function of the two-dimensional Fourier transform $Y=\operatorname{fft} 2(X, m, n)$ for the $(m \times n)$-matrix $X$, which allows the sums like

$Y_{p+1, q+1}=\sum_{j=0}^{m-1} \sum_{k=0}^{n-1} X_{j+1, k+1} \mathrm{e}^{-2 \pi i(j p / M+k q / N)}$ to be calculated very efficiently and quickly. As a result, we obtain a two-dimensional matrix for the values of the complex variable $Y$ with the same dimensions as the matrix $X$. Then it is necessary to calculate the matrix $H(m, n)=|Y(m, n)|^{2}$. After that, the radial averaging of the matrix $H(m, n)$ is performed to obtain a one-dimensional function $H(q)$ in which the wave vector is calculated using the formula

$q=\frac{2 \pi j}{\min (m, n) \Delta}$,

where $\min (m, n)$ is the minimum of the $m$ - and $n$-values, $\Delta$ is the horizontal distance between the neighbor points in the data file with the altitude values, and $j$ is an integer number varying from 1 to $[\min (m, n) / 2-1]$. If the linear size of the system (the side of the square) equals $L$, the minimum and maximum values of the wave vector $\mathbf{q}$ are given as follows: $q_{\min }=2 \pi / L$ and $q_{\max }=\pi \min (m, n) / L$. Finally, we calculate the one-dimensional function $C(q)$ using the following formula:

$C(q)=\frac{H(q) \Delta^{2}}{(2 \pi)^{2} \min (m, n)^{2}}$.

The procedure for the spectral density calculation, which was described above, makes it possible to find the function $C(q)$ that coincides with definition (22). The analysis of the function $C(q)$ is a modern and very wide-spread method to examine rough fractal surfaces, which are very often formed in various experiments (see, e.g., works [30-32]) owing to internal self-organization processes. If a surface possesses fractal properties, then, with the help of the formula [21]

$C(q)=C_{0}\left(\frac{q}{q_{0}}\right)^{-2(1+H)}$,

the classical Hurst exponent $H$ which is associated with the fractal dimension of the surface $D_{f}=3-H$ can be calculated.

In our case, according to the images shown in Fig. 6, it is reasonable to assume that fractal properties can be attributed to the distributions calculated for the parameters $D_{u S}=D_{v S}=10^{-3}$ and exhibited in the upper panels of the figure. The calculations carried out for those surfaces, however, testify that the corresponding distributions do not have fractal characteristics. However, it is almost always 
fractal objects that are formed in nature in various self-organization processes. This fact evidences that a running self-organizing process (which can undoubtedly be described by the Lorentz system) is not a sufficient condition for the appearance of fractal properties. Some authors say that random factors may play an important role in the formation of fractal properties $[27,33]$. Their action can be described by considering the stochastic terms in the Lorentz equations, which we intend to do in our future works.

\section{Conclusions}

We numerically studied the Lorentz system of equations with gradient terms describing the spatial distribution of basic parameters, being taken into account in each equation. It is found that three principally different modes can arise, depending on the degree of influence by the gradient contributions. In the first case, the mode of deterministic chaos is realized similarly to the original system. However, the spatial distribution of the order parameter permanently varies at that and does not evolve to a certain stationary distribution. In the second case, a slowly evolving distribution of the order parameter, which is close to the stationary state, is established in time. As a result, the system becomes divided into approximately two equal parts with positive and negative order parameter values (however, such a proportional distribution can be realized only under certain initial conditions). Finally, in the third mode, a rapid homogenization of the system takes place, at which the order parameter acquires the same value over the entire simulation area, and the system demonstrates a homogeneous mode which reproduces the classical Lorenz attractor. In the future, we plan to perform similar studies of the Lorentz system making allowance for fluctuations which can substantially change the behavior of a dynamic system, as it occurs, e.g., in the shear-induced melting model [29].

This work was carried out in the framework of the state budget theme No. 0117U003923 of the Ministry of Education and Science of Ukraine (20172020). The work was also partially supported by the Ministry of Education and Science of Ukraine (the grant of the President of Ukraine to support scientific researches of young scientists "Transitional mode between adhesion and sliding in tangential dynamic contact for normal and gradient materials", competition
F82 of the State Fund for Fundamental Research of Ukraine, the state registration No. 0119U103175).

1. H. Haken. Synergetics: An Introduction. Nonequilibrium Phase Transition and Self-Organization in Physics, Chemistry, and Biology (Springer, 1978) [ISBN: 978-3-64296469-5].

2. A.V. Voronov, V.M. Petnikova, V.V. Shuvalov. "Magnetodipole" self-organization of charge carriers in high- $T_{c}$ superconductors and the kinetics of phase transition. J. Exp. Theor. Phys. 93, 1091 (2001).

3. R. Meucci, F. Salvadori, K.A.M. Al Naimee et al. Attractor selection in a modulated laser and in the Lorenz circuit. Philos. Trans. Royal Soc. A 366, 475 (2008).

4. S.E. Boulfelfel, A.R. Oganov, S. Leoni. Understanding the nature of "superhard graphite". Sci. Rep. 2, 47 (2012).

5. L. Yong, Z.X-. Fang, B.Q-. Sheng. Synchronization and control of autocatalytic chemical reaction in continuous stirring tank reactor. Acta Phys. Sin. 57, 4748 (2008).

6. V.I. Zasimchuk, E.E. Zasimchuk, A.S. Gatsenko. Selforganization in viscous fluids. Metallofiz. Noveish. Tekhnol. 39, 1435 (2017) (in Russian).

7. H. Haken. Information and Self-Organization. A Macroscopic Approach to Complex Systems (Springer, 2006) [ISBN: 978-3-540-33023-3].

8. Y. Holovatch, R. Kenna, S. Thurner. Complex systems: physics beyond physics. Eur. J. Phys. 38, 023002 (2017).

9. E.N. Lorenz. Deterministic nonperiodic flow. J. Atmos. Sci. 20, 130 (1963).

10. I.A. Shuda, S.S. Borysov, A.I. Olemskoi. Noise-induced oscillations in non-equilibrium steady state systems. Phys. Scr. 79, 065001 (2009).

11. A.I. Olemskoi, O.V. Yushchenko, T.I. Zhilenko. Investigation of conditions for a self-organized transition to the bistable regime of quasi-equilibrium condensation and stripping of the surface. Phys. Solid State 53, 845 (2011).

12. A.I. Olemskoi, A.V. Khomenko. Three-parameter kinetics of phase transition. Zh. Èksp. Teor. Fiz. 110, 2144 (1996) (in Russian).

13. A.I. Olemskoi, A.V. Khomenko, V.P. Koverda. Explosive crystallization of ultradisperse amorphous film. Physica $A$ 284, 79 (2000).

14. A.I. Olemskoi, A.V. Khomenko, D.O. Kharchenko. Selforganized criticality within fractional Lorenz scheme. Physica A 323, 263 (2003).

15. I.A. Lyashenko, N.N. Manko. Synergetic model of boundary friction taking into account spatial nonuniformity of stresses, strain, and temperature. Tech. Phys. 59, 1737 (2014).

16. Ya.A. Lyashenko. Formation of heterogeneous spatial structures in a boundary lubrication layer during friction. J. Appl. Mech. Tech. Phys. 57, 136 (2016).

17. A.A. Samarskii, A.V. Gulin. Stability of Difference Schemes (Nauka, 1973) (in Russian). 
18. G.E.P. Box, M.E. Muller. A note on the generation of random normal deviates. Ann. Math. Statist. 29, 610 (1958).

19. J.W. Kantellhardt, S.A. Zschiegner, E. Koscielny-Bunde et al. Multifractal detrended fluctuation analysis of nonstationary time series. Physica A 316, 87 (2002).

20. O.I. Olemskoi, S.M. Danyl'chenko, V.M. Borysyuk, I.O. Shuda. Multifractal analysis of X-ray patterns of complex condensed media. Metallofiz. Noveish. Tekhnol. 31, 777 (2009).

21. B.N.J. Persson. On the fractal dimension of rough surfaces. Tribol. Lett. 54, 99 (2014).

22. E.S. Gadelmawla, M.M. Koura, T.M.A. Maksoud, I.M. Elewa, H.H. Soliman. Roughness parameters. J. Mater. Process. Technol. 123, 133 (2002).

23. Q. Li, R. Pohrt, V.L. Popov. Adhesive strength of contacts of rough spheres. Front. Mech. Eng. 5, 1 (2019).

24. Q. Li, R. Pohrt, I.A. Lyashenko, V.L. Popov. Boundary element method for nonadhesive and adhesive contacts of a coated elastic half-space. Proc. Inst. Mech. Eng. J. 234 (1), 73 (2019).

25. K.L. Johnson. The adhesion of two elastic bodies with slightly wavy surfaces. Int. J. Solids Struct. 32, 423 (1995).

26. Z.M. Makukha, S.I. Protsenko, L.V. Odnodvorets, I.Yu. Protsenko. Magneto-strain effect in double-layer film systems. J. Nano-Electron. Phys. 4, 02043 (2012).

27. A.E. Filippov, S.N. Gorb. Methods of the pattern formation in numerical modeling of biological problems. $F U$ Mech. Eng. 17, 217 (2019).

28. A.I. Dmitriev, A.Yu. Nikonov, W. Osterle, B.Ch. Jim. Verification of Rabinowicz' criterion by direct molecular dynamics modeling. FU Mech. Eng. 17, 207 (2019).

29. I.A. Lyashenko, V.N. Borysiuk, N.N. Manko. Statistical analysis of self-similar behaviour in the shear induced melting model. Condens. Matter Phys. 17, 23003 (2014).

30. V. Perekrestov, V. Latyshev, A. Kornyushchenko, Y. Kosminska. Formation, charge transfer, structural and morphological characteristics of $\mathrm{ZnO}$ fractal-percolation nanosystems. J. Electron. Mater. 48, 2788 (2019).

31. V.M. Latyshev, V.I. Perekrestov, A.S. Kornyushchenko, I.V. Zahaiko. Formation of porous zinc nanosystems using direct and reverse flows of DC magnetron sputtering. Funct. Mater. 24, 154 (2017).

32. A.S. Kornyushchenko, V.V. Natalich, V.I. Perekrestov. Formation of copper porous structures under near-equilibrium chemical vapor deposition. J. Cryst. Growth 442, 68 (2016).

33. A.D. Pogrebnjak, V.N. Borisyuk, A.A. Bagdasaryan, O.V. Maksakova, E.V. Smirnova. The multifractal investigation of surface microgeometry of (Ti-Hf-Zr-V-Nb)N nitride coatings. J. Nano-Electron Phys. 6, 04018 (2014).

Reseived 17.09.19.

Translated from Ukrainian by O.I. Voitenko

З.М. Ляшенко, Я.О. Ляшенко

ВПЛИВ ПРОСТОРОВОї

НЕОДНОРІДНОСТІ НА ФОРМУВАННЯ ХАОТИЧНИХ РЕЖИМІВ ПРОЦЕСУ САМООРГАНІЗАЦІЇ

$\mathrm{P}$ е $з$ ю м е

Чисельно розв'язано систему рівнянь Лоренца з урахуванням градієнтних доданків. Розглянуто три принципово різні режими. У першому режимі просторовий розподіл параметра порядку еволюціонує в часі, утворюючи домени двох типів з додатним і від'ємним значенням параметра порядку. У другому - розподіл близький до стаціонарного. I у третьому режимі по всьому простору параметр порядку приймає однакові значення. Для перших двох випадків розраховано залежності середньої площі доменів, їх кількості та сумарної площі від часу. В останньому випадку внесок градієнтних доданків повністю нівелюється і реалізується класичний аттрактор Лоренца. 